\title{
High expression of GALNT7 promotes invasion and proliferation of glioma cells
}

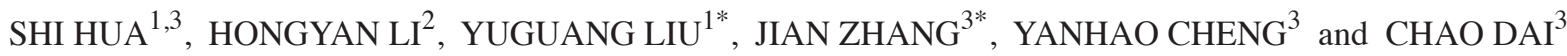 \\ ${ }^{1}$ Department of Neurosurgery, Qilu Hospital of Shandong University, Brain Science Research Institute of \\ Shandong University, Jinan, Shandong $250012 ;{ }^{2}$ Central Laboratory, and ${ }^{3}$ Minimally Invasive and \\ Functional Department of Neurosurgery, Linyi People's Hospital of Shandong University, \\ Linyi, Shandong 276000, P.R. China
}

Received February 7, 2018; Accepted July 20, 2018

DOI: $10.3892 / 01.2018 .9498$

\begin{abstract}
Polypeptide-N-acetyl-galactosaminlytransferase 7 (GALNT7), a member of the GalNAc-transferase family, has not been previously evaluated as a prognostic factor of glioblastoma (GBM) or low-grade glioma (LGG). Based on The Cancer Genome Atlas database and bioinformatics analyses, the expression of GALNT7 was demosntrated to be higher in GBM and LGG tissues than in normal brain tissue. The expression levels of GANLT7 were associated with age, tumor grade, survival rate, disease-free survival time and overall survival time. Gene correlation and gene-set enrichment analyses suggested that GALNT7 may affect the
\end{abstract}

Correspondence to: Dr Yuguang Liu, Department of Neurosurgery, Qilu Hospital of Shandong University, Brain Science Research Institute of Shandong University, NB107 Wenhua West Road, Jinan, Shandong 250012, P.R. China

E-mail:252900349@qq.com

Dr Jian Zhang, Minimally Invasive and Functional Department of Neurosurgery, Linyi People's Hospital of Shandong University, Lanshan, NB130 Yizhou Road, Lanshan, Linyi, Shandong 276000, P.R. China

E-mail: honest20699@126.com

*Contributed equally

Abbreviations: GALNT7, polypeptide-N-acetyl-galactosam inlytransferase 7; TCGA, The Cancer Genome Atlas; KEGG, Kyoto Encyclopedia of Genes and Genomes database; GTEx, Genotype-Tissue Expression project; GBM, glioblastoma; LGG, low grade glioma; NOM P-val, nominal P-values; FDR, false discovery rates; MsigDB, Molecular Signatures Database; NK, natural killer; CAMS, cell adhesion molecules; CNS, central nervous system; MIF, macrophage migration inhibitory factor; JAK, janus kinase; STAT, signal transducer and activator of transcription; GSEA, gene-set enrichment analysis

Key words: polypeptide-N-acetyl-galactosaminlytransferase 7, glioma, The Cancer Genome Atlas, Kyoto Encyclopedia of Genes and Genomes, survival, analysis, pathway, prognosis proliferative and invasive abilities of glioma cells through multiple signaling pathways, including regulation of the actin cytoskeleton, natural killer cell-mediated cytotoxicity, the janus kinase-signal transducer and activator of transcription (STAT) signaling pathway, cell adhesion molecules and extracellular matrix receptor interaction pathways. Furthermore, 5 target genes of GALNT7 involved in these signaling pathways were identified, including Crk, Rac family small GTPase 1, STAT3, poliovirus receptor and Tenascin C. In summary, high expression of GALNT7 was associated with poor prognosis of glioma, and may be used as an effective biomarker of glioma.

\section{Introduction}

Glioma is the most common and aggressive type of tumor of the human central nervous system (CNS). The annual incidence of glioma has been reported as 30 80/1 million worldwide (1). Regarding pathological grade, glioma includes glioblastoma (GBM) and low-grade glioma (LGG). Currently, standard treatments for glioma include chemoradiotherapy and targeted therapy, which are administered following maximum surgical resection (2-5). The prognosis of glioma was reported to have a 5-year survival rate of $\sim 10-20 \%$ (6). Therapeutic techniques for increasing the survival rate and improving quality of life of patients with glioma in the neurosurgical field $(7,8)$.

Polypeptide-N-acetyl-galactosaminlytransferase 7 (GALNT7) is a member of the N-acetyl-D-galactosaminetransferase family. The enzyme encoded by this gene controls the initiation step of mucin-type O-linked protein glycosylation and transfers $\mathrm{N}$-acetyl galactosamine to serine and threonine amino acid residues (9). Previous studies have indicated that GALNT7 can influence the prognoses of multiple types of malignant tumor, including cervical (10), esophageal (11) and liver cancer (12), as well as renal cell carcinoma (13), by affecting cell proliferation, metastasis, apoptosis, migration, differentiation and invasion (14-17).

The Cancer Genome Atlas (TCGA) is a publicly funded project that aims to catalogue and identify major cancer-causing genomic alterations, to create a comprehensive 'atlas' of genomic profiles of various types of cancer. To date, the 
program has investigated $>30$ types of human tumor, including GBM and LGG, through large-scale genome sequencing and integrated multi-dimensional analyses (18). To the best of our knowledge, the present study is the first to investigate the role of GALNT7 in glioma, using GBM and LGG RNA-seq data downloaded from the TCGA database.

\section{Materials and methods}

Data collection. The RNA-seq expression and clinical data of 174 patients with GBM and 529 patients with LGG were obtained from TCGA (using the search terms: GBM and LGG, level 3 and RNA-seq; https://cancergenome.nih.gov/). Complete gene expression data, clinical data and definite pathological diagnosis was available for all included patients. RNA-seq expression data for 114 normal brain tissues were download from the GTEx project (https://www.gtexportal. org/home/; Brain-Cortex-GTEx, RNA-seq) and used as the control group (19).

Statistical analysis. SPSS 21.0 (IBM Corp., Armonk, NY, USA) was used for data processing. Quantitative data are expressed as the mean \pm standard deviation $(\mathrm{x} \pm \mathrm{s})$. Two samples were compared by t-test and multiple comparisons were performed by one-way analysis of variance followed by Least-Significant-Difference test. Qualitative data were expressed as a percentage (\%) and analyzed by $\chi^{2}$. Survival analysis was performed using Cox regression and Kaplan-Meier analysis $(\mathrm{P}<0.05)$. Gene-set enrichment analysis was performed using GSEA (version, 2.2.1; http://software.broadinstitute. org/gsea/downloads.jsp) from the Molecular Signatures Database (v6.3 MsigDB; Broad Institute, Inc., Massachusetts Institute of Technology, and Regents of the University of California). According to default-weighted enrichment, the cut-offs for the number of random combinations was 1,000, for nominal P-values (NOM P-val) $<0.05$, and false discovery rates (FDR), $<0.25$. Pearson correlation coefficient was used to assess the association between the expression of 2 genes. $\mathrm{P}<0.05$ was considered to indicate a statistically significant difference.

\section{Results}

GALNT7 expression is positively associated with glioma malignancy. GALNT7 expression levels were significantly higher in glioma, particularly in GBM, compared with the negative control samples $(\mathrm{P}<0.05)$. This indicates a positive association between GALNT7 expression and glioma malignancy (Table I; Fig. 1A).

Association between GALNT7 expression and clinical characteristics. GALNT7 was expressed at different levels in normal brain tissues and glioma tissues. In order to investigate the effect of its expression in glioma, GALNT7 expression in normal brain tissue from GTEx was employed as the cut-off value, and TCGA data regarding expression of GALNT7 in glioma tissue were divided into high (HIGH) and low (LOW) groups. The association between GALNT7 expression levels and clinical characteristics of patients with glioma was analyzed using t-test. The results demonstrated that expression
Table I. Differential analysis of GLANT7 RNA-seq expression.

\begin{tabular}{lcccc}
\hline Group & No. & $\begin{array}{c}\text { mean } \pm \text { standard } \\
\text { deviation }\end{array}$ & F & P-value \\
\hline GBM & 174 & $3.46 \pm 1.38$ & 15.37 & $<0.05$ \\
LGG & 529 & $2.92 \pm 1.58$ & & \\
Normal & 114 & $2.46 \pm 1.58$ & & \\
\hline
\end{tabular}

GBM, glioblastoma; LGG, low-grade glioma. F, one-way ANOVA value.

level was not associated with sex, but it was associated with age, tumor grade and survival rate (Table II).

Negative association between GALNT7 expression and survival time. Using the median GALNT7 expression value as the standard, GALNT7 expression data were divided into HIGH GALNT7 transcripts per million (TPM) and LOW GALNT7 TPM groups. Cox survival analysis demonstrated a regression coefficient (B) of $0.102(\mathrm{P}<0.05$; Table III). Together with Kaplan-Meier survival analysis, this suggests that patients with glioma exhibiting high expression of GALNT7 have relatively shorter disease-free (Fig. 1B) and overall (Fig. 1C) survival times than those exhibiting low expression.

GSEA and prediction of co-expressed genes. Based on the KEGG database (20), the results of GSEA (NOM P-val $<0.05$; FDR $<0.25$ ) indicated 6 pathways associated with glioma, including 'regulation of the actin cytoskeleton' (Fig. 2A), 'natural killer cell-mediated cytotoxicity' (Fig. 2B), 'JAK-STAT signaling' (Fig. 2C), 'cell adhesion molecules' ('CAMs') (Fig. 2D) and 'ECM-receptor interaction' (Fig. 2E). In order to predict genes that are co-expressed, Pearson's correlation analysis was used to determine the correlation between GALNT7 expression and all genes associated with these signaling pathways. As a result, Crk (Fig. 2F), RAC1 (Fig. 2G), STAT3 (Fig. 2H), PVR (Fig. 2I) and Tnc (Fig. 2J) were identified as potential target genes. Crk participates in regulating the 'actin cytoskeleton' pathway, $\mathrm{RACl}$ is involved in the 'natural killer cell mediated cytotoxicity' pathway and STAT3 is associated with the 'JAK-STAT' signaling pathway. Also, PVR participates in the 'CAMs' pathway and Tnc is involved in the 'ECM-receptor interaction' pathway.

Differential and survival analysis of co-expressed genes in glioma. Differential analyses demonstrated that Crk (Fig. 3A) expression is positively associated with the malignancy of glioma; positive association was also observed with RAC1 (Fig. 3B), STAT3 (Fig. 3C), and Tnc (Fig. 3E). PVR (Fig. 3D) highly expressed in GBM, but expressed lower than normal brain tissue in LGG $(\mathrm{P}<0.05)$. Survival analyses demonstrate that disease-free time was negatively correlated with the expression of RAC1 (Fig. 4B), STAT3 (Fig. 4C), PVR (Fig. 4D) and Tnc (Fig. 4E), but not Crk (Fig. 4A). Meanwhile, the expression levels of Crk (Fig. 4F), RAC1 (Fig. 4G), STAT3 (Fig. 4H), PVR (Fig. 4I) and Tnc (Fig. 4J) were negatively correlated with overall survival time $(\mathrm{P}<0.05)$. 
Table II. Clinical characteristics of patients with glioma.

\begin{tabular}{|c|c|c|c|c|c|}
\hline Characteristics & No. & Low GALNT7 expression (\%) & High GALNT7 expression (\%) & $\chi^{2}$ & P-value \\
\hline Age (years) & & & & 10.46 & $<0.05$ \\
\hline$<40$ & 274 & $132(48.2)$ & $142(51.8)$ & & \\
\hline$\geq 42$ & 414 & $149(36.0)$ & $265(64.0)$ & & \\
\hline Sex & & & & 0.05 & 0.82 \\
\hline Male & 393 & $162(41.2)$ & $231(58.8)$ & & \\
\hline Female & 295 & $119(40.3)$ & $176(59.7)$ & & \\
\hline Grade & & & & 38.37 & $<0.05$ \\
\hline $\mathrm{G} 2$ & 257 & $138(53.7)$ & 119 (46.3) & & \\
\hline G3 & 263 & $104(39.5)$ & $159(60.5)$ & & \\
\hline G4 & 168 & $39(23.2)$ & $129(76.8)$ & & \\
\hline Survival status & & & & 38.456 & $<0.05$ \\
\hline Not alive & 416 & $209(50.2)$ & 207 (49.8) & & \\
\hline Alive & 272 & $72(26.5)$ & $200(73.5)$ & & \\
\hline
\end{tabular}

GLANT7, polypeptide-N-acetyl-galactosaminlytransferase 7. Part of the follow up data from TCGA was missing, including 6 patients with GBM and 9 patients with LGG. Therefore these patients were excluded from form Table II.

Table III. Cox regression analysis of GALNT7 expression.

\begin{tabular}{lccccccc}
\hline GALNT7 & B & SE & Wald & df & P-value & HR & 95\% Confidence intervals \\
\hline 0.10 & 0.02 & 30.94 & 1 & $<0.05$ & 1.11 & $(1.07,1.15)$
\end{tabular}

GLANT7, polypeptide-N-acetyl-galactosaminlytransferase 7; B, regression coefficient; SE, standard error; Wald, $\chi^{2}$ value; df, degree of freedom; HR, hazard ratio.
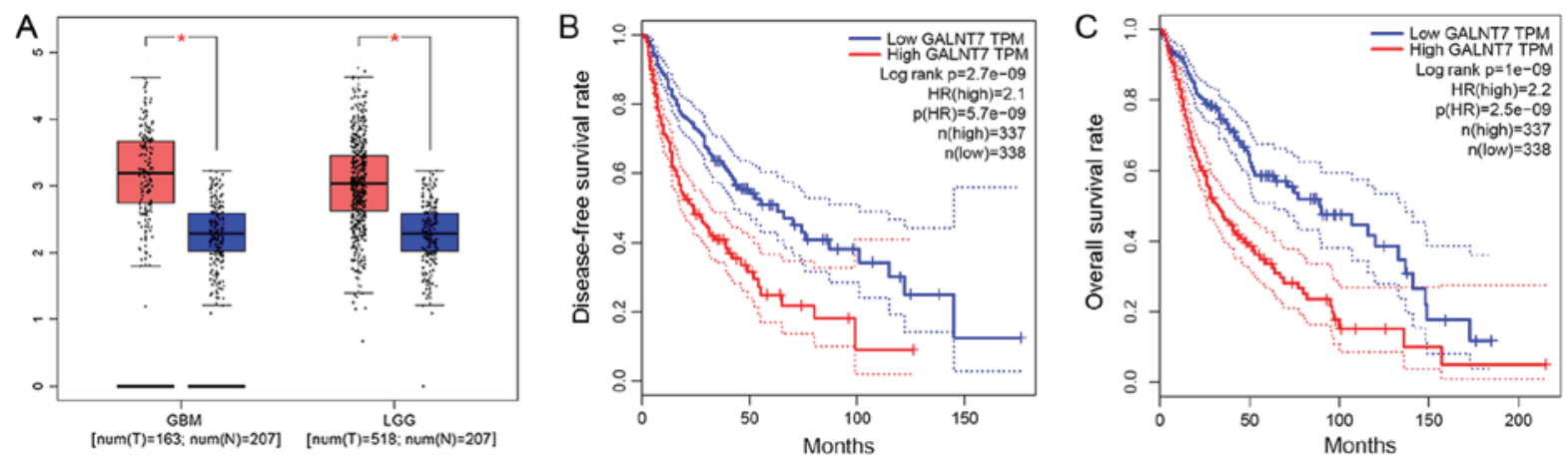

Figure 1. Differential expression and survival analysis of GLANT7 in glioma. (A) Tumor tissue, red; normal tissue, blue. GLANT7 expression was higher in GBM and LGG than in normal brain tissue. Cox regression and Kaplan-Meier analyses suggest that patients with glioma exhibiting high expression of GALNT7 had relatively shorter (B) disease-free and (C) overall survival times. "P $<0.05$. GLANT7, polypeptide-N-acetyl-galactosaminlytransferase 7; GBM, gliblastoma; LGG, low-grade glioma; TPM, transcripts per million.

\section{Discussion}

Glioma is divided into 4 grades according to the World Health Organization standard (1): Grade I, pilocytic astrocytoma, which manifests as a benign tumor and patients may have a full recovery following total tumor resection; Grade II, includes oligoastrocytoma and diffuse astrocytoma, it has a poorer prognosis compared with Grade I, but is still considered to be LGG (2); Grade III, includes anaplastic astrocytoma, and
Grade IV, GBM. Grade III and IV tumors are associated with high degrees of malignancy, strong invasive abilities, poor prognosis and multiple differentiation potentials $(3,21)$. The present study aimed to reveal the association between GALNT7 expression and glioma by identifying co-expressed genes and relevant signaling pathways.

Firstly, a positive correlation between GALNT7 expression and the malignancy of glioma was assessed by analyzing TCGA glioma datasets. Cox and Kaplan-Meier survival analyses 

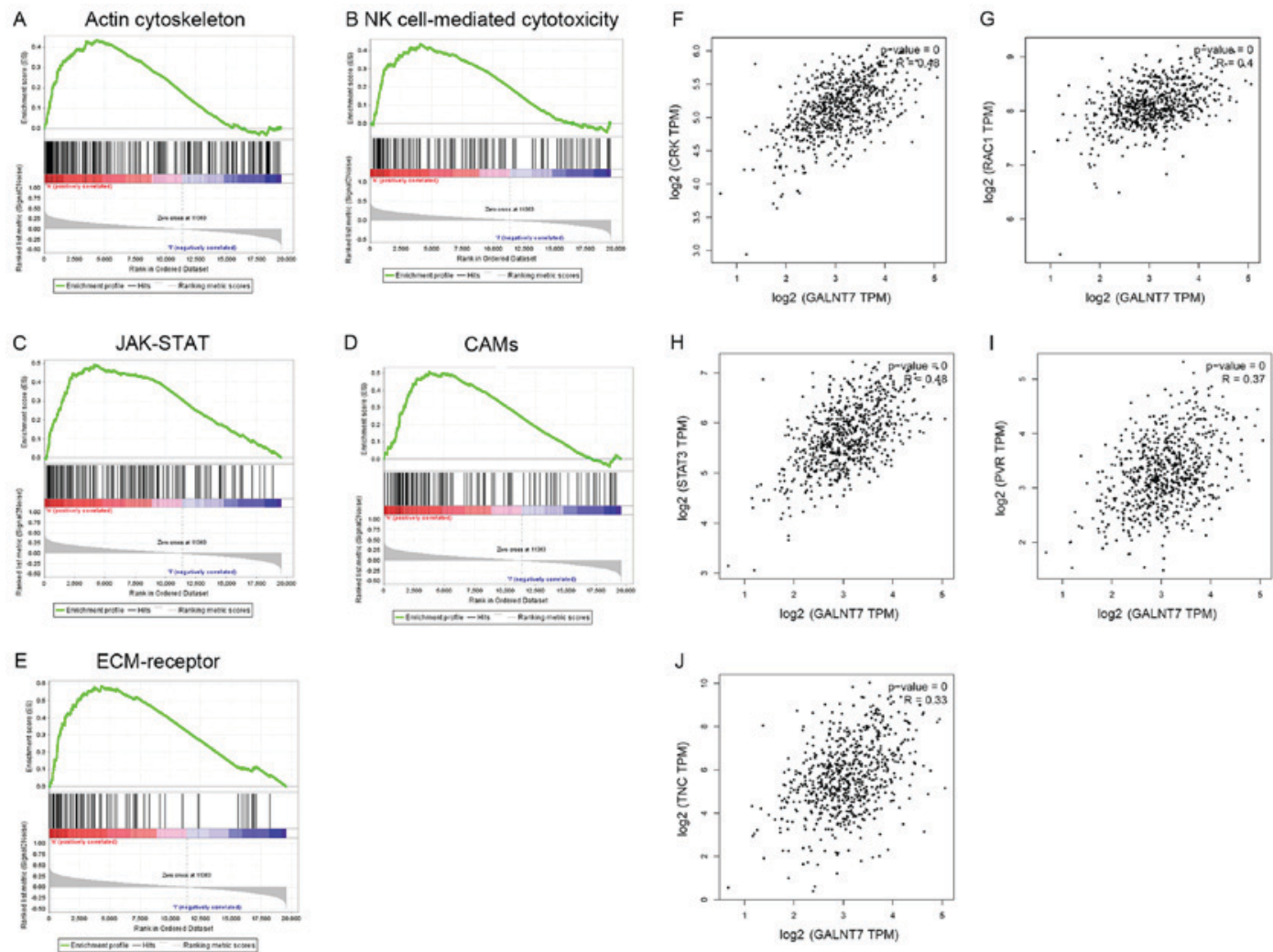

Figure 2. Gene-set enrichment analysis and co-expression prediction. The results of GSEA (Nominal P-value $<0.05$, false discovery rate $<0.25$ ) indicated six pathways up-regulated by GALNT7 and associated with glioma, including (A) 'regulation of actin cytoskeleton', N (B) 'natural killer cell-mediated cytotoxicity', (C) JAK-STAT signaling pathway, (D) 'Cell adhesion molecules', (E) 'ECM-receptor interaction'. The potential target genes identified by Pearson's correlation coefficient included (F) 'Crk', (G) RAC1, (H) STAT3, (I) PVR, and (J) Tnc (P<0.05). Crk functions in the 'regulation of actin cytoskeleton' pathway, RAC1 functions in the 'natural killer cell mediated cytotoxicity' pathway, STAT3 functions in the 'JAK-STAT signaling' pathway, PVR functions in the 'Cell adhesion molecules' pathway, and Tnc functions in the 'ECM-receptor interaction' pathway. GSEA, gene set enrichment analysis; GALNT7, polypeptide-N-acetyl-galactosaminlytransferase 7; JAK, janus kinase; STAT, signal transducer and activator of transcription; ECM, extracellular matrix; PVR, poliovirus receptor; Tnc, Tenascsin C.
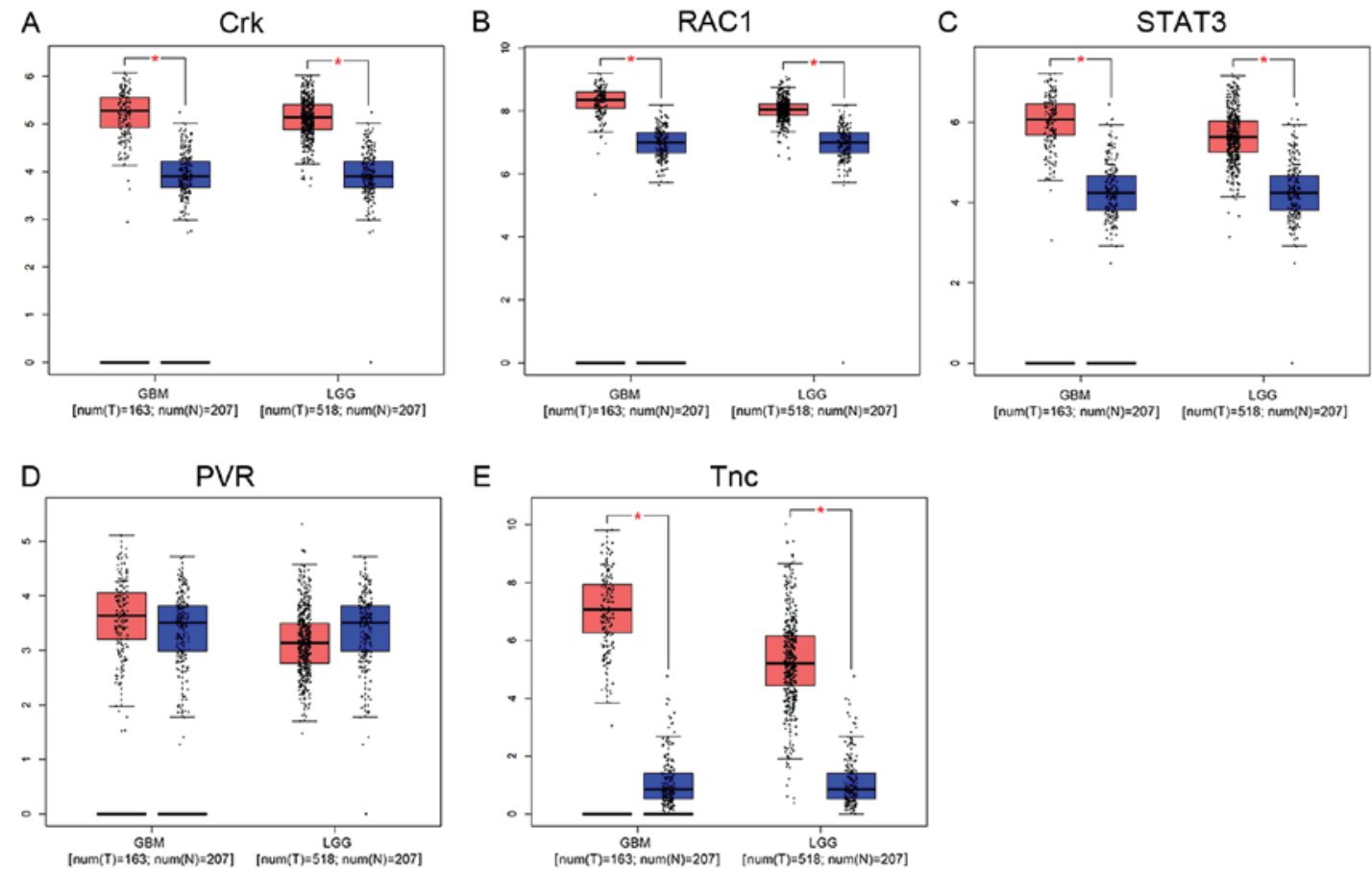

Figure 3. Differential analysis of co-expressed genes in glioma. Tumor tissue, red; normal tissue, blue. Differential analyses revealed that (A) Crk, (B) RAC1, (C) STAT3 and (E) Tnc expression was positively associated with the malignancy of glioma. (D) PVR was highly expressed in GBM, but expressed at a lower level than in normal brain tissue in LGG $(\mathrm{P}<0.05)$. RAC1, Rac family amall GTPase 1; STAT3, signal transducer and activator of transcription 3; Tnc, Tenascsin 3; PVR, poliovirus receptor; GBM, glioblastoma; LGG, low-grade glioma. 

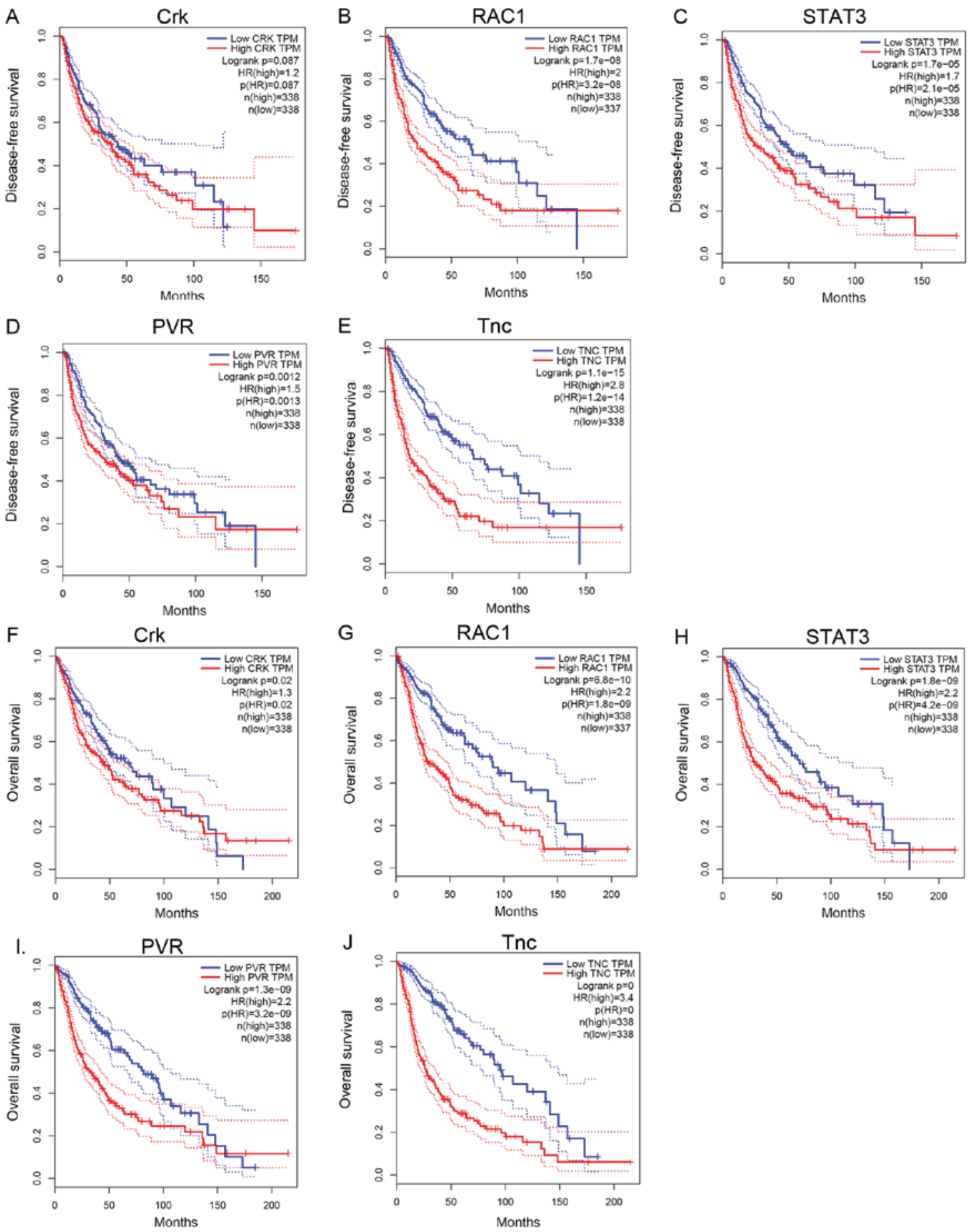

Figure 4. Survival analysis of co-expressed genes in glioma. (A) Kaplan-Meier survival analysis revealed that the expression of Crk was not associated with disease-free survival time (P>0.05), whereas that of (B) RAC1, (C) STAT3, (D) PVR, and (E) Tnc were negatively associated with disease-free survival time $(\mathrm{P}<0.05)$. Overall survival time was negatively associated with the expression of (F) Crk, (G) RAC1, (H) STAT3, (I) PVR and (J) Tnc (P<0.05). RAC1, Rac family amall GTPase 1; STAT3, signal transducer and activator of transcription 3; PVR, poliovirus receptor; Tnc, Tenascsin 3.

demonstrated that patients with glioma exhibiting high expression levels of GALNT7 had relatively short disease-free and overall survival times. Therefore, high expression levels of GALNT7 may affect the prognosis of glioma.

Correlation analyses suggested that Crk, RAC1, STAT3, PVR and Tnc are genes that are co-expressed with GALNT7. Survival and differential analyses demonstrated that the expression of Crk, RAC1, STAT3, PVR and Tnc are positively associated with the malignancy of glioma, and are negatively associated with survival time. These results were consistent with those of previous studies, which indicated that high expression levels of Crk, RAC1, STAT3, PVR and Tnc were associated with poor prognosis by promoting the invasion, proliferation and other biological functions of glioma (22-27). Therefore, we speculate that GALNT7 may affect the progression of glioma by upregulating the expression of target genes through relevant signaling pathways. Based on this hypothesis, GSEA $(28,29)$ was used to identify such relevant signaling 
pathways. As a result, 5 signaling pathways associated with GALNT7 and glioma were identified, including 'regulation of the actin cytoskeleton', 'natural killer cell-mediated cytotoxicity', 'JAK-STAT signaling', 'CAMs' and 'ECM-receptor interaction' pathways. Specific target genes of GALNT7 participate in each signaling pathway, and were subsequently evaluated.

There are a variety of intracellular actin binding proteins that regulate multiple cellular functions, including changes in the structure of the actin cytoskeleton, which occur through the binding or dissociating of the proteins with actin (30). Regulation of the actin cytoskeleton pathway governs the cell motility of glioma by regulating the locomotion of the cytoskeleton, and slows the recovery of patients with glioma $(31,32)$. Crk participates in regulation of the 'actin cytoskeleton pathway', and its expression is associated with overall survival time of patients with glioma. Tsuda et al (22) demonstrate that overexpression of Crk can increase the invasive potential of cancer cells by notably inducing tyrosine phosphorylation of scaffolding molecules, including p130 (Cas) and paxillin through Src family tyrosine kinases, and stimulating the activation loop of intracellular signaling. Therefore, as a gene that is co-expressed with Crk, GALNT7 promotes the invasion of glioma by upregulating Crk expression through regulation of the actin cytoskeleton pathway.

NK cells form the first line of immune defense against tumors $(33,34)$. Such cells destroy tumors by controlling cell proliferation, cytotoxicity and cytokine production in the early stages of tumor formation $(35,36)$. RAC1 has been reported to be involved in the NK cell-mediated cytotoxicity pathway (37). Former research suggests that high expression levels of RAC1 inhibit the cytotoxic effects of NK cells by two mechanisms: Decreased interaction between NK and target cells; NK cells that do interact have a reduced ability to polarize their effector molecules towards target cells (38). Based on these results, coexpression with RAC1 and high expression of GALNT7 inhibits the cytotoxic effects of NK cells by regulating the natural killer cell-mediated cytotoxicity pathway, which leads to loss of control of glioma cell proliferation and poor prognosis.

The JAK-STAT signaling pathway is mainly formed of the tyrosine kinase associated receptor, JAK and STAT (39). Previous studies reported that the activation of the JAK-STAT signaling pathway is associated with poor prognosis of glioma $(40,41)$. Additionally, a previous study verified that high expression levels of STAT3 enhance the proliferation of glioma cells (24). In the present study, GALNT7 was coexpressed with STAT3, and high expression levels of STAT3 were associated with poor prognosis of glioma. This suggests that GALNT7 may trigger and increase in glioma cell proliferation by activating the JAK-STAT signaling pathway through upregulation of STAT3 expression.

CAMs are membrane and transmembrane glycoproteins that regulate intercellular, cell and extracellular matrix interactions, which are closely associated with cell adhesion, migration, differentiation and signal transduction $(42,43)$. CAMs are widely expressed in glioma and are regulated by the CAMs signaling pathway (44). PVR is involved in the CAMs signaling pathway, and can enhance the metastasis of glioblastoma through its over-expression (45). As GALNT7 is co-expressed with PVR, GALNT7 may regulate the CAMs signaling pathway by up-regulating PVR, thereby influencing tumor cell migration and affecting prognosis.

ECM is mainly formed of insoluble components that contribute to the behavior and structure of stromal cells and epithelial vessels, respectively. The ECM constitutes collagen, elastin, proteoglycan and glycoprotein. ECM can influence cell differentiation, proliferation, adhesion, morphogenesis and phenotypic expression (46). Brösicke et al $(47,48)$ proposed Tnc as a key gene in the ECM-receptor interaction pathway and that high coexpression with GALNT7 occurs within glioma tissue, which is consistent with our research. Accumulating evidence also suggests that Tnc serves a crucial role in cell migration and invasion, the most malignant characteristics of glioma. Therefore, GALNT7 can affect the ECM-receptor interaction pathway by upregulating the expression of Tnc; this mechanism may affect the invasion of glioma cells and lead to a poor prognosis of glioma.

In conclusion, high expression of GALNT7 was demonstrated to be associated with poor prognosis of glioma, likely via promoting invasion and proliferation through multiple signaling pathways. Therefore, GALNT7 may be employed as a novel molecular target for the early detection, diagnosis and treatment of glioma.

\section{Acknowledgements}

Not applicable.

\section{Funding}

No funding was received.

\section{Availability of data and materials}

The datasets generated or analyzed during the current study are available in the TCGA/KEGG/GTEx repository, [https://cancergenome.nih.gov/] [https://www.kegg.jp/] [https://www.gtexportal.org/home/].

\section{Authors' contributions}

SH contributed to writing the manuscript, and was responsible for project design and data interpretation; YC and CD were responsible for data collection and preliminary analysis; HL was responsible for all data analyses; YL and JZ conceived and designed the present study given final approval of the version to be published.

\section{Ethical approval and consent to participate}

Not applicable.

\section{Patient consent for publication}

Not applicable.

\section{Competing interests}

The authors declare that they have no competing interests. 


\section{References}

1. Louis DN, Perry A, Reifenberger G, von Deimling A, Figarella-Branger D, Cavenee WK, Ohgaki H, Wiestler OD, Kleihues P and Ellison DW: The 2016 world health organization classification of tumors of the central nervous system: A summary. Acta Neuropathol 131: 803-820, 2016.

2. Forst DA, Nahed BV, Loeffler JS and Batchelor TT: Low-grade gliomas. Oncologist 19: 403-413, 2014.

3. de Groot JF: High-grade gliomas. Continuum (Minneap Minn.) 21: 332-344, 2015.

4. Kim IA, Yang YJ, Yoon SC, Choi IB, Kay CS, Kwon HC, Kim CM, Joe YA, Kang JK and Hong YK: Potential of adenoviral p53 gene therapy and irradiation for the treatment of malignant gliomas. Int J Oncol 19: 1041-1047, 2001.

5. Maris D, Nica D, Mohan D, Moisa $H$ and Ciurea AV: Multidisciplinary management of adult low grade gliomas. Chirurgia (Bucur) 109: 590-599, 2014.

6. Pekmezci M and Perry A: Genetic markers in adult high-grade gliomas. Semin Radiat Oncol 24: 235-239, 2014.

7. Duffy A, Le J, Sausville E and Emadi A: Autophagy modulation: A target for cancer treatment development. Cancer Chemother Pharmacol 75: 439-447, 2015.

8. Lin Q, Mao KL, Tian FR, Yang JJ, Chen PP, Xu J, Fan ZL, Zhao YP, Li WF, Zheng L, et al: Brain tumor-targeted delivery and therapy by focused ultrasound introduced doxorubicin-loaded cationic liposomes. Cancer Chemother Pharmacol 77: 269-280, 2016.

9. Bennett EP, Hassan H, Hollingsworth MA and Clausen H: A novel human UDP-N-acetyl-D-galactosamine: Polypeptide $\mathrm{N}$-acetylgalactosaminyltransferase, GalNAc-T7, with specificity for partial GalNAc-glycosylated acceptor substrates. FEBS Lett 460: 226-230, 1999.

10. Wu H, Chen J, Li D, Liu X, Li L and Wang K: MicroRNA-30e functions as a tumor suppressor in cervical carcinoma cells through targeting GALNT7. Transl Oncol 10: 876-885, 2017.

11. Lu Q, Xu L, Li C, Yuan Y, Huang S and Chen H: miR-214 inhibits invasion and migration via downregulating GALNT7 in esophageal squamous cell cancer. Tumour Biol 37: 14605-14614, 2016.

12. Shan SW, Fang L, Shatseva T, Rutnam ZJ, Yang X, Du W, Lu WY, Xuan JW, Deng Z and Yang BB: Mature miR-17-5p and passenger miR-17-3p induce hepatocellular carcinoma by targeting PTEN, GalNT7 and vimentin in different signal pathways. J Cell Sci 126: 1517-1530, 2013.

13. Li Y, Li Y, Chen D, Jin L, Su Z, Liu J, Duan H, Li X, Qi Z, Shi M, et al: miR30a5p in the tumorigenesis of renal cell carcinoma: A tumor suppressive microRNA. Mol Med Rep 13: 4085-4094, 2016.

14. Duan HF, Li XQ, Hu HY, Li YC, Cai Z, Mei XS, Yu P, Nie LP, Zhang W, Yu ZD and Nie GH: Functional elucidation of miR-494 in the tumorigenesis of nasopharyngeal carcinoma. Tumour Biol 36: 6679-6689, 2015.

15. Li W, Ma H and Sun J: MicroRNA34a/c function as tumor suppressors in Hep2 laryngeal carcinoma cells and may reduce GALNT7 expression. Mol Med Rep 9: 1293-1298, 2014.

16. Gaziel-Sovran A, Segura MF, Di Micco R, Collins MK, Hanniford D, Vega-Saenz de Miera E, Rakus JF, Dankert JF, Shang S, Kerbel RS, et al: miR-30b/30d regulation of GalNAc transferases enhances invasion and immunosuppression during metastasis. Cancer Cell 20: 104-118, 2011.

17. Mayer A, Schneider F, Vaupel P, Sommer C and Schmidberger H: Differential expression of HIF-1 in glioblastoma multiforme and anaplastic astrocytoma. Int J Oncol 41: 1260-1270, 2012

18. Gonda DD, Cheung VJ, Muller KA, Goyal A, Carter BS and Chen CC: The cancer genome atlas expression profiles of low-grade gliomas. Neurosurg Focus 36: E23, 2014.

19. The Genotype-Tissue Expression (GTEx) project. Nat Genet 45 : 580-585, 2013.

20. Du J, Yuan Z, Ma Z, Song J, Xie X and Chen Y: KEGG-PATH: Kyoto encyclopedia of genes and genomes-based pathway analysis using a path analysis model. Mol Biosyst 10: 2441-2447, 2014.

21. Pouleau HB, Sadeghi N, Baleriaux D, Melot C, De Witte O and Lefranc F: High levels of cellular proliferation predict pseudoprogression in glioblastoma patients. Int J Oncol 40: 923-928, 2012.

22. Tsuda M and Tanaka S: Roles for crk in cancer metastasis and invasion. Genes Cancer 3: 334-340, 2012.
23. Yukinaga H, Shionyu C, Hirata E, Ui-Tei K, Nagashima T, Kondo S, Okada-Hatakeyama M, Naoki $\mathrm{H}$ and Matsuda $\mathrm{M}$ : Fluctuation of Rac1 activity is associated with the phenotypic and transcriptional heterogeneity of glioma cells. J Cell Sci 127: 1805-1815, 2014.

24. Priester M, Copanaki E, Vafaizadeh V, Hensel S, Bernreuther C, Glatzel M, Seifert V, Groner B, Kögel D and Weissenberger J: STAT3 silencing inhibits glioma single cell infiltration and tumor growth. Neuro Oncol 15: 840-852, 2013.

25. Chandramohan V, Bryant JD, Piao H, Keir ST, Lipp ES Lefaivre M, Perkinson K, Bigner DD, Gromeier $M$ and McLendon RE: Validation of an Immunohistochemistry Assay for Detection of CD155, the poliovirus receptor, in malignant gliomas. Arch Pathol Lab Med 141: 1697-1704, 2017.

26. Xia S, Lal B, Tung B, Wang S, Goodwin CR and Laterra J: Tumor microenvironment tenascin-C promotes glioblastoma invasion and negatively regulates tumor proliferation. Neuro Oncol 18: 507-517, 2016.

27. Brosicke N, van Landeghem FK, Scheffler B and Faissner A: Tenascin- $\mathrm{C}$ is expressed by human glioma in vivo and shows a strong association with tumor blood vessels. Cell Tissue Res 354: 409-430, 2013.

28. Machado CM, Freitas AT and Couto FM: Enrichment analysis applied to disease prognosis. J Biomed Semantics 4: 21, 2013

29. Mueller C, deCarvalho AC, Mikkelsen T, Lehman NL, Calvert V, Espina V, Liotta LA and Petricoin EF III: Glioblastoma cell enrichment is critical for analysis of phosphorylated drug targets and proteomic-genomic correlations. Cancer Res 74: 818-828, 2014.

30. Hou M, Liu X, Cao J and Chen B: SEPT7 overexpression inhibits glioma cell migration by targeting the actin cytoskeleton pathway. Oncol Rep 35: 2003-2010, 2016.

31. Klopocka W, Korczynski J and Pomorski P: Cytoskeleton and nucleotide signaling in glioma C6 cells. Adv Exp Med Biol 986: 103-119, 2013.

32. Uzdensky A, Kristiansen B, Moan J and Juzeniene A: Dynamics of signaling, cytoskeleton and cell cycle regulation proteins in glioblastoma cells after sub-lethal photodynamic treatment: Antibody microarray study. Biochim Biophys Acta 1820: 795-803, 2012.

33. Huang BY, Zhan YP, Zong WJ, Yu CJ, Li JF, Qu YM and Han S: The PD-1/B7-H1 pathway modulates the natural killer cells versus mouse glioma stem cells. PLoS One 10: e0134715, 2015.

34. Miller JS: Biology of natural killer cells in cancer and infection. Cancer Invest 20: 405-419, 2002

35. He W, Kuang Y, Xing X, Simpson RJ, Huang H, Yang T, Chen J, Yang L, Liu E, He W and Gu J: Proteomic comparison of 3D and 2D glioma models reveals increased HLA-E expression in $3 \mathrm{D}$ models is associated with resistance to NK cell-mediated cytotoxicity. J Proteome Res 13: 2272-2281, 2014.

36. Orozco-Morales M, Sanchez-Garcia FJ, Golan-Cancela I, Hernández-Pedro N, Costoya JA, de la Cruz VP, Moreno-Jiménez S, Sotelo J and Pineda B: RB mutation and RAS overexpression induce resistance to NK cell-mediated cytotoxicity in glioma cells. Cancer Cell Int 15: 57, 2015.

37. Mainiero F, Soriani A, Strippoli R, Jacobelli J, Gismondi A, Piccoli M, Frati L and Santoni A: RAC1/P38 MAPK signaling pathway controls betal integrin-induced interleukin-8 production in human natural killer cells. Immunity 12: 7-16, 2000.

38. Billadeau DD, Brumbaugh KM, Dick CJ, Schoon RA, Bustelo XR and Leibson PJ: The Vav-Racl pathway in cytotoxic lymphocytes regulates the generation of cell-mediated killing. J Exp Med 188: 549-559, 1998.

39. Nicolas CS, Amici M, Bortolotto ZA, Doherty A, Csaba Z, Fafouri A, Dournaud P, Gressens P, Collingridge GL and Peineau S: The role of JAK-STAT signaling within the CNS. JAKSTAT 2: e22925, 2013

40. Tu Y, Zhong Y, Fu J, Cao Y, Fu G, Tian X and Wang B: Activation of JAK/STAT signal pathway predicts poor prognosis of patients with gliomas. Med Oncol 28: 15-23, 2011.

41. Zheng Q, Han L, Dong Y, Tian J, Huang W, Liu Z, Jia X, Jiang T, Zhang J, Li X, et al: JAK2/STAT3 targeted therapy suppresses tumor invasion via disruption of the EGFRvIII/JAK2/STAT3 axis and associated focal adhesion in EGFRvIII-expressing glioblastoma. Neuro Oncol 16: 1229-1243, 2014.

42. Zhao WJ and Schachner M: Neuregulin 1 enhances cell adhesion molecule 11 expression in human glioma cells and promotes their migration as a function of malignancy. J Neuropathol Exp Neurol 72: 244-255, 2013. 
43. Li J, Liu X, Duan Y, Liu Y, Wang H, Lian S, Zhuang G and Fan Y: Combined blockade of T cell immunoglobulin and mucin domain 3 and carcinoembryonic antigen-related cell adhesion molecule 1 results in durable therapeutic efficacy in mice with intracranial gliomas. Med Sci Monit 23: 3593-3602, 2017.

44. Chen X, Ma WY, Xu SC, Liang Y, Fu YB, Pang B, Xin T, Fan HT, Zhang R, Luo JG, et al: The overexpression of epithelial cell adhesion molecule (EpCAM) in glioma. J Neurooncol 119: 39-47, 2014

45. Sloan KE, Stewart JK, Treloar AF, Matthews RT and Jay DG: CD155/PVR enhances glioma cell dispersal by regulating adhesion signaling and focal adhesion dynamics. Cancer Res 65 : 10930-10937, 2005

46. Brösicke N and Faissner A: Role of tenascins in the ECM of gliomas. Cell Adh Migr 9: 131-140, 2015.
47. Brosicke N, Sallouh M, Prior LM, Job A, Weberskirch R and Faissner A: Extracellular matrix glycoprotein-derived synthetic peptides differentially modulate glioma and sarcoma cell migration. Cell Mol Neurobiol 35: 741-753, 2015.

48. Shimizu T, Kurozumi K, Ishida J, Ichikawa T and Date I: Adhesion molecules and the extracellular matrix as drug targets for glioma. Brain Tumor Pathol 33: 97-106, 2016.

c) (1) (9) This work is licensed under a Creative Commons (c) Attribution-NonCommercial-NoDerivatives 4.0 International (CC BY-NC-ND 4.0) License. 